\title{
Anterior Transsternal Approach for Treatment of Upper Thoracic Vertebral Osteomyelitis: Case Report and Review of the Literature
}

\author{
Hai V. Le ${ }^{1}$, Rishi Wadhwa ${ }^{2}$, Praveen Mummaneni ${ }^{2}$, Pierre Theodore ${ }^{3}$ \\ 1. Orthopedic Surgery, Massachusetts General Hospital 2. Department of Neurological Surgery, UCSF \\ Medical Center 3. Department of Thoracic Surgery, UCSF Medical Center
}

$\square$ Corresponding author: Hai V. Le, hle6@partners.org

Disclosures can be found in Additional Information at the end of the article

\section{Abstract}

Direct ventral access to the cervicothoracic spine (C7-T4) poses a technical challenge in spine surgery, given the vital neurovascular structures residing anterior to the cervicothoracic junction (CTJ). The transsternal approach is a feasible surgical option that allows for direct anterior exposure of the lower cervical and upper thoracic vertebrae. Here, the authors report a case of an elderly gentleman with upper thoracic (T1-2) vertebral osteomyelitis and epidural abscess who underwent a transsternal full median sternotomy for ventral decompression and fusion of C7-T2. We also detail our operative procedure and review relevant literature on different transsternal approaches to the CTJ.

Categories: General Surgery, Neurosurgery, Orthopedics

Keywords: transsternal approach, osteomyelitis, cervicothoracic junction, epidural abscess, median sternotomy, spine surgery

\section{Introduction}

Obtaining direct ventral access to the cervicothoracic spine (C7-T2) for decompression and fusion is technically challenging, given the anatomical constraints. Operative exposure of the cervicothoracic junction (CTJ) is obscured by the skeleton of the thorax (i.e., sternum, clavicles, and ribs). The anterior transsternal approach is a feasible surgical option that allows for direct exposure of the anterior vertebral elements of the CTJ. With a transsternal approach, there are many vital neurovascular structures close by, so great care needs to be carried out while dissecting away the vascular compartment of the superior mediastinum to achieve adequate exposure. Anatomical structures at risk for injury include structures within the carotid sheath, trachea, esophagus, recurrent laryngeal nerves, great vessels, vertebral arteries, and sympathetic trunk [1-6].

Here, the authors report a case of an elderly gentleman with upper thoracic vertebral osteomyelitis (T1-2) and epidural abscess who underwent a transsternal full median sternotomy surgical approach for ventral decompression and fusion of C7-T2. We also detail our operative procedure and review relevant literature on different transsternal approaches to the CTJ.

\section{Case Presentation}




\section{Cureus}

Informed patient consent was obtained for this patient's treatment.

\section{History}

The patient was a 68-year-old diabetic gentleman with a recent history of methicillin-resistant Staphylococcus aureus (MRSA) bacteremia secondary to pneumonia, managed with one course of antibiotics. He presented to the emergency department (ED) from an outside hospital for neck pain. In the ED, the patient was afebrile with normal vital signs. Physical exam was within normal limit except for pain on palpation at the neck and right deltoid. Sensory and motor exam were intact. He had an initial WBC count of 13.5, and blood cultures were MRSA positive. Preoperative CT imaging showed erosions of the T1 and T2 vertebral bodies with loss of the intervertebral disc space, consistent with discitis and osteomyelitis. There was evidence of prominent paravertebral soft tissue abnormality, compatible with extension of infection into adjacent soft tissues (Figure 1).
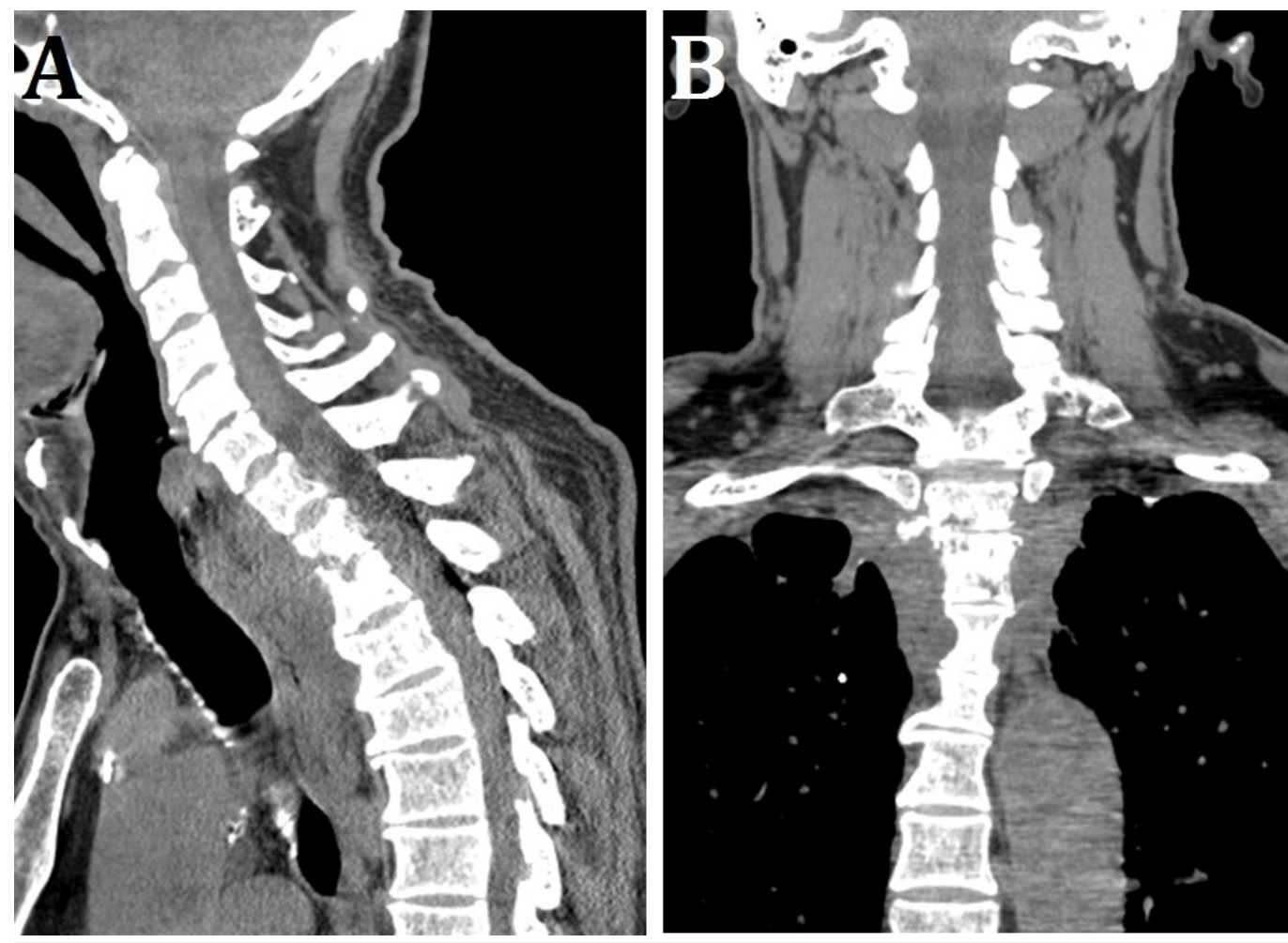

\section{FIGURE 1: Preoperative CT Imaging}

Preoperative (A) sagittal and (B) coronal CT imaging showing erosions of the T1 and T2 vertebral bodies, with loss of the intervertebral disc space, consistent with discitis and osteomyelitis.

Preoperative MRI showed discitis and osteomyelitis at T1 and T2, anterior epidural collection extending from C7-T2, and severe canal stenosis and cord compression (Figure 2). 


\section{Cureus}
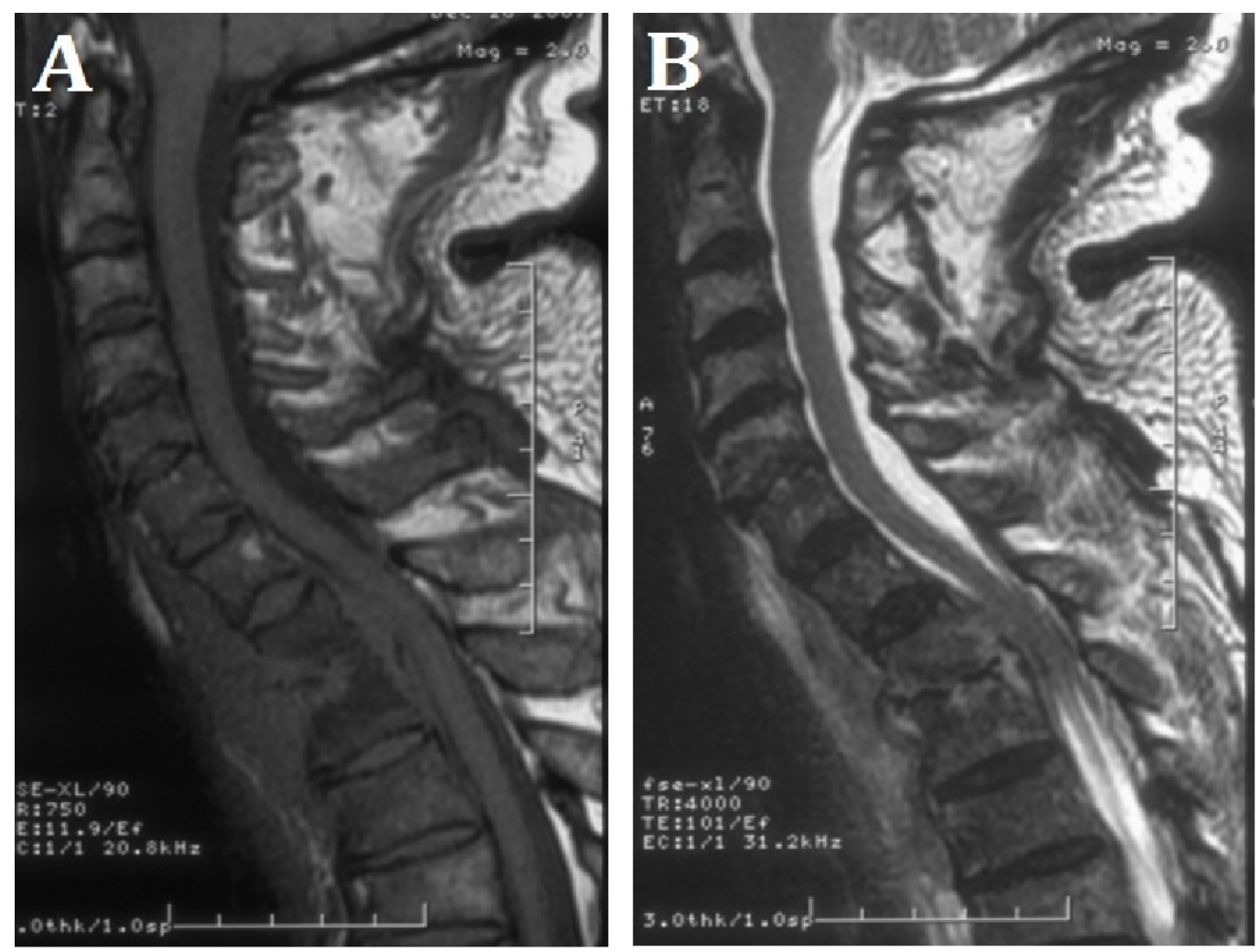

\section{FIGURE 2: Preoperative MRI Imaging}

Preoperative sagittal (A) T1- and (B) T2-weighted MRI showing discitis and osteomyelitis at T1 and T2, anterior epidural collection extending from C7-T2, and severe canal stenosis and cord compression.

The patient was diagnosed with T1 and T2 osteomyelitis with an epidural abscess causing cord compression. Vancomycin and gentamicin were started, and Neurosurgery Service was consulted for surgical decompression.

\section{Operation}

The patient was taken to the operating room the next day. Under general anesthesia, the patient was appropriately prepped, draped, and positioned supine. Prophylactic antibiotics were administered. Thoracic Surgery (P.T.) performed the initial transsternal exposure of the cervicothoracic spine. A linear incision on the medial border of the right sternocleidomastoid muscle was made and extended down over the manubrium. Median sternotomy was performed with the sternal saw. The sternocleidomastoid was distracted laterally, and the trachea and esophagus were distracted medially for exposure of the anterior cervicothoracic spine. Next, Neurological Surgery (P.M.) performed T1 corpectomy, T2 partial corpectomy, and C7-T2 anterior interbody arthrodesis and plate fixation. Fluoroscopy was utilized to identify the C7-T2 vertebrae. The C7-T1 and T1-T2 disc spaces were incised, and the discs were removed with pituitary rongeurs and curettes. The T1 and T2 vertebral bodies were noted to be partially collapsed, with hollow cavities and covered with phlegmon. The T1 and the upper $50 \%$ of the $\mathrm{T} 2$ vertebral bodies were removed. An epidural collection of phlegmon was identified and evacuated. Phlegmon adherent to the dura was carefully removed using microdissection techniques. The thecal sac was freed up, and the spinal cord from C7-T2 was decompressed. Foraminotomies were also performed. Next, the surgical site was thoroughly irrigated with fluids containing vancomycin and gentamycin. A mesh cage filled with autograft bone marrow 


\section{Cureus}

from the sternum was tamped into position to create an anterior arthrodesis and fusion from C7-T2. A cervical plate was drilled and tapped in place using $5 \mathrm{~mm}$ variable screws. Hemostasis was achieved with the bipolar, and a Jackson-Pratt drain was placed. Closure of the sternum was performed with No. 6 sternal wires, and the skin closure was performed in the normal fashion. There was no intraoperative complication and estimated blood loss (EBL) was $500 \mathrm{cc}$.

\section{Postoperative course}

The patient tolerated the above procedure well. Postoperative CT imaging showed interval partial corpectomy of the $\mathrm{T} 1$ and $\mathrm{T} 2$ vertebral bodies with anterior spinal fusion extending from C7 through T2 vertebral bodies (Figure 3).
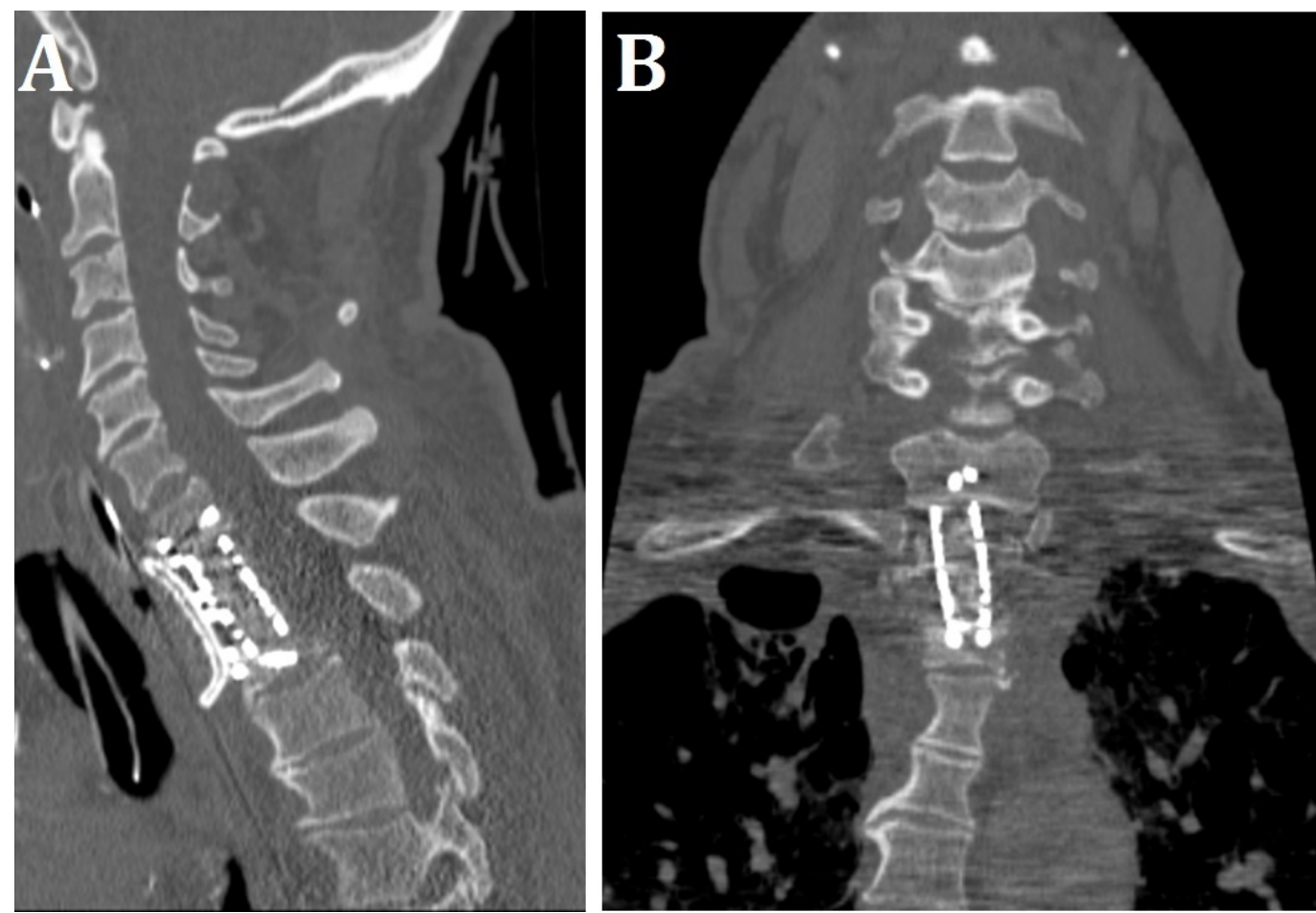

\section{FIGURE 3: Postoperative CT Imaging}

Postoperative (A) sagittal and (B) coronal CT imaging showing interval partial corpectomy of T1 and $\mathrm{T} 2$ vertebral bodies with anterior spinal fusion extending from $\mathrm{C} 7$ through $\mathrm{T} 2$ vertebral bodies.

Surgical biopsies showed dense plasmacytic infiltrate consistent with osteomyelitis.

\section{Discussion}

As clearly illustrated in this case example, the transsternal full median sternotomy approach offers adequate direct exposure of the lower cervical and upper thoracic vertebrae for surgical treatment of pathology affecting the cervicothoracic spine. Injury to vital neurovascular structures can be avoided with meticulous dissection of the vascular compartment of the superior mediastinum by the thoracic surgeon. Vital structures should be correctly identified, tagged, and gently retracted prior to operating on the spine.

There have been several other clinical studies validating the feasibility of the transsternal approach. Jiang, et al. (2010) evaluated 16 patients who underwent limited sternotomy with a 
transverse sternal split for the treatment of upper thoracic vertebral tuberculosis (TB). All of the patients tolerated the procedure well and improved postoperatively from a neurological standpoint. There was no incidence of hardware failure or TB recurrence, and the mean time to spinal bone fusion was 4.2 months [7]. Zengming, et al. (2010) studied 54 patients with upper thoracic disease (33 cases of TB, 14 cases of neoplasm, five cases of eosinophilic granuloma, and two cases of traumatic fracture) who underwent anterior decompression and fusion with full median sternotomy. Pain resolved in all of the patients, and motor deficits were improved in patients who initially presented with radiculopathy or myelopathy. There was no serious postoperative approach-related complication [8].

The transsternal approach presented in this article utilizes a full median sternotomy for access of the cervicothoracic spine. However, there have been many modifications to this approach in order to limit extensive osteotomy, such as manubriotomy with clavicle resection, partial lateral manubriotomy, and partial sternotomy with a transverse sternal split [3, 7, 9-13]. There are several potential advantages of a full median sternotomy approach. First of all, it is a technically simpler procedure compared to other modified approaches. Second, it offers better exposure of the mediastinum for improved visualization and manipulation of important neurovascular structures to avoid intraoperative complications. Third, the pectoral girdle is preserved since there is no resection of the clavicle. Lastly, extension of the operative field caudally to as low as $\mathrm{T} 5$ can be achieved by dissecting a plane between the brachiocephalic vein, superior vena cava, and ascending aorta [3].

\section{Conclusions}

The transsternal approach utilizing a full median sternotomy can be a safe and effective surgical approach to directly access pathology affecting the lower cervical and upper thoracic vertebrae. Compared to other modified transsternal approaches, the full median sternotomy approach is technically simpler, provides better exposure, preserves the pectoral girdle, and allows for extension of the operative field caudally.

\section{Appendices}

\section{Additional Information Disclosures}

Human subjects: Consent was obtained by all participants in this study. Conflicts of interest: In compliance with the ICMJE uniform disclosure form, all authors declare the following:

Payment/services info: All authors have declared that no financial support was received from any organization for the submitted work. Financial relationships: All authors have declared that they have no financial relationships at present or within the previous three years with any organizations that might have an interest in the submitted work. Other relationships: All authors have declared that there are no other relationships or activities that could appear to have influenced the submitted work.

\section{References}

1. Le H, Thongtrangan I, Kim DH: Surgical anatomy and approaches to the cervicothoracic junction. Spinal Instrumentation: Surgical Techniques. Kim DH, Vaccaro AR, Fessler RG (ed): Thieme, New York, NY; 2005. 3-13.

2. Brau SA: Anterior approach to the cervicothoracic spine. Surgical Approaches to the Spine. 
Watkins RG III, Watkins RG IV (ed): Springer, New York, NY; 2003. 69-72.

3. Maciejczak A, Radek A, Kowalewski J, Palewicz A: Anterior transsternal approach to the upper thoracic spine. Acta Chir Hung. 1999, 38:83-86.

4. Calliauw L, Dallenga A, Caemaert J: Trans-sternal approach to intraspinal tumours in the upper thoracic region. Acta Neurochir (Wien). 1994, 127:227-31. 10.1007/BF01808771

5. Charles R, Govender S: Anterior approach to the upper thoracic vertebrae. J Bone Joint Surg Br. 1989, 71:81-84.

6. Miscusi M, Bellitti A, Polli FM: Surgical approaches to the cervico-thoracic junction. J Neurosurg Sci. 2005, 49:49-57.

7. Jiang H, Xiao ZM, Zhan XL, He ML: Anterior transsternal approach for treatment of upper thoracic vertebral tuberculosis. Orthop Surg. 2010, 2:305-9. 10.1111/j.1757-7861.2010.00104.X

8. Zengming X, Maolin H, Xinli Z, Qianfen C: Anterior transsternal approach for a lesion in the upper thoracic vertebral body. J Neurosurg Spine. 2010, 13:461-68. 10.3171/2010.4.SPINE09808

9. Tubbs RS, Loukas M, Callahan JD, Cohen-Gadol AA: A novel approach to the upper anterior thoracic spine: a cadaveric feasibility study. J Neurosurg Spine. 2010, 13:346-50. 10.3171/2010.3.SPINE09814

10. Lesoin F, Thomas CE 3rd, Autricque A, Villette L, Jomin M: A transsternal biclavicular approach to the upper anterior thoracic spine. Surg Neurol. 1986, 26:253-56. 10.1016/00903019(86)90158-8

11. Sundaresan N, Shah J, Feghali JG: A transsternal approach to the upper thoracic vertebrae . Am J Surg. 1984, 148:473-77. 10.1016/0002-9610(84)90372-6

12. Sundaresan N, Shah J, Foley KM, Rosen G: An anterior surgical approach to the upper thoracic vertebrae. J Neurosurg. 1984, 61:686-90. 10.3171/jns.1984.61.4.0686

13. Tamura M, Saito M, Machida M, Shibasaki K: A transsternoclavicular approach for the anterior decompression and fusion of the upper thoracic spine. Technical note. J Neurosurg Spine. 2005, 2:226-29. 10.3171/spi.2005.2.2.0226 